\title{
Temporal and spatial strategies in an active place avoidance task on Carousel: A study of effects of stability of arena rotation speed
}

Stepan Bahnik, Ales Stuchlik

The active place avoidance task is a dry-arena task used to assess spatial navigation and memory in rodents. In this task, a subject is put on a rotating circular arena and avoids an invisible sector that is stable in relation to the room. Rotation of the arena means that the subject's avoidance must be active, otherwise the subject will be moved in the to-beavoided sector by the rotation of the arena and a slight electric shock will be administered. The present experiment explored the effect of variable arena rotation speed on the ability to avoid the to-be-avoided sector. Subjects in a group with variable arena rotation speed learned to avoid the sector with the same speed and attained the same avoidance ability as rats in a group with a stable arena rotation speed. Only a slight difference in preferred position within the room was found between the two groups. No difference was found between the two groups in the dark phase, where subjects could not use orientation cues in the room. Only one rat was able to learn the avoidance of the to-be-avoided sector in this phase. The results of the experiment suggest that idiothetic orientation and interval timing are not crucial for learning avoidance of the to-be-avoided sector. However, idiothetic orientation might be sufficient for avoiding the sector in the dark. 
1 Temporal and spatial strategies in an active place avoidance task on 2 Carousel: A study of effects of stability of arena rotation speed ${ }^{1}$

3

4 Štěpán Bahník ${ }^{1,2^{*}}$, Aleš Stuchlík ${ }^{1}$

5

$6 \quad{ }^{1}$ Department of Neurophysiology of Memory, Institute of Physiology, Academy of Sciences of

7 the Czech Republic, Prague, Czech Republic

$8 \quad 2$ Department of Psychology, University of Würzburg, Würzburg, Germany

9

$10 *$ Corresponding author

11 Email: bahniks@seznam.cz (ŠB)

${ }^{1}$ The experiment described in the present paper was part of the master's thesis of the first author. 
12 Abstract

13 The active place avoidance task is used to assess spatial navigation and memory in rodents. In this dry-arena task, a subject is put on a rotating circular arena and avoids an invisible sector that is stable in relation to the room. Rotation of the arena means that the subject's avoidance must be active, otherwise the subject will be moved in the to-be-avoided sector by the rotation of the arena and a slight electric shock will be administered. The present experiment explored the effect of variable arena rotation speed on the ability to avoid the to-be-avoided sector. Subjects in a group with variable arena rotation speed learned to avoid the sector with the same speed and attained the same avoidance ability as rats in a group with a stable arena rotation speed. Only a slight difference in preferred position within the room was found between the two groups. No difference was found between the two groups in the dark phase, where subjects could not use orientation cues in the room. Only one rat was able to learn the avoidance of the to-be-avoided sector in this phase. The results of the experiment suggest that idiothetic orientation and interval timing are not crucial for learning avoidance of the to-beavoided sector. However, idiothetic orientation might be sufficient for avoiding the sector in the dark.

Keywords

spatial navigation, interval timing, substratal idiothetic navigation, inertial idiothetic navigation, rats

\section{Introduction ${ }^{2}$}

The active place avoidance task (APA; formerly also called active allothetic place avoidance) [17] is a variant of a place avoidance task [1] used for assessing spatial navigation and memory in rodents. The task uses a dry and smooth circular arena made of metal (Carousel) which contains an unmarked to-be-avoided sector (usually a $60^{\circ}$ section of the arena), entering which is punished by a mild footshock. It can be used both for rats [6, 8-9] and mice [10-11]. The tobe-avoided sector is stable within the room and the arena rotates on its axis in the active version of the task. This means that successful performance requires active avoidance of the tobe-avoided sector, e.g. to walk in the direction opposite to arena rotation. Since the sector is not directly visible, the subject has to remember its location in relation to orientation cues outside of the rotating arena, i.e. in the experiment room. Therefore, efficient avoidance of the sector requires continual locomotion [7] and spatial navigation [3]. The task and special modifications to it (such as two-frame place avoidance) were used in various domains of animal

\footnotetext{
${ }^{2}$ Abbreviations: APA: active place avoidance, rpm: revolution per minute
} 
cognition studies such as in pharmacological [12-13], lesion [14], genetic [15-16], and electrophysiological studies [17]. Notably, the task has been extensively employed in studies focused on animal models of neuropsychiatric disorders, involving schizophrenia [8, 18-19], ischemia [20], and traumatic brain injury [21-22]. Thus, the task has a high pre-clinical significance, despite the fact that only a few laboratories worldwide use it at this time. Therefore, attempts to provide deeper insight into learning mechanisms involved in performance in the task are of great importance [23-24].

This paper focuses on the examination of possible strategies to solve the task. The standard variant of the active place avoidance task on the Carousel uses a stable speed of arena rotation - usually 1 revolution per minute (rpm). One of the possible strategies of avoidance then is to move with the same average speed against the direction of arena rotation. In order to use this method of avoidance, a subject does not need to use allothetic orientation (using external spatial cues) [25]. Use of idiothetic orientation (based on information generated by locomotion of an animal) [26], possibly with interval timing, i.e. perception of time at intervals ranging from seconds to minutes [27-28], would be sufficient in this case [29-30]. A subject may thus move continuously against the direction of arena rotation with the same average speed as the speed of arena rotation, or move against the direction of the rotation with a certain periodicity across the distance which regulates position of the subject within a room. If subjects, at least partly, use this avoidance strategy, the interpretation of results of the studies using the APA task would have to take into account that possible performance deficits may be caused by impairment in idiothetic orientation or interval timing. Interval timing is known to be influenced by drugs [31] and its deficits are seen in neuropathology [32], which are both areas where the APA task is used [6].

The present study used the manipulation of arena rotation speed to influence relevance of temporal information for successful avoidance in the APA task. Variable rotation speed should influence the possibility of using a combination of inertial idiothesis (i.e. use of information from internal organs sensing movement, such as proprioceptors, vestibular apparatus, and proprioception) [33] and interval timing for avoidance of the to-be-avoided sector. On the other hand, in the case of stable arena rotation speed a subject can move with a certain frequency across distance needed for regulation of its position. For example, a subject on an arena with the stable rotation speed $1 \mathrm{rpm}$ can move every $30 \mathrm{~s}$ by $180^{\circ}$ to avoid the to-be-avoided sector. In the case of variable rotation speed, the subject has no such opportunity because the required distance and frequency of movement for regulation of its position within a room necessarily varies depending on rotation speed. Additionally, the use of inertial idiothesis may be further affected if rotation speed changes not only within a session but between sessions as well. In that case, a subject may not easily "learn the speed" required to regulate its position within a room. On the other hand, it is possible that variable arena rotation speed may 
influence attention given to inertial stimuli by making the rotation speed relevant for avoidance of the to-be-avoided sector and therefore making it salient for the subject, thus leading to better performance in the task. The present study allowed us to explore these possibilities.

\section{Method}

\section{Ethics statement}

This study was carried out in strict accordance with Animal Protection Code of Czech republic, EU directive 2010/63/EC and with the recommendations in the Guide for the Care and Use of Laboratory Animals of the National Institutes of Health. The protocol including needle implantation described in the Subjects section was approved by the Committee on the Ethics of Animal Experiments of the Institute of Physiology Academy of Sciences of the Czech Republic (Permit Number: 136/2013). No surgery was performed, and all efforts were made to minimize suffering of animals.

\section{Subjects}

The experiment was conducted with 16 male Long-Evans rats obtained from the breeding colony of the Institute of Physiology, Academy of Sciences of the Czech Republic. At the beginning of the experiment, the rats were 16-17 weeks old and had mean weight $415 \mathrm{~g}$ (SD = $27 \mathrm{~g})$. They were housed in transparent plastic $25 \times 30 \times 40 \mathrm{~cm}$ boxes in an air-conditioned animal room with a stable temperature and $12 / 12 \mathrm{~h}$ light/dark cycle. All parts of the experiment were conducted during the light phase of the day. Water and food pellets were available ad libitum throughout the study. Prior to behavioral tests, rats were implanted with hypodermic needle through skin fold between shoulders. The needle was used for attaching an alligator clip delivering electric shocks throughout the experiment. The implantation procedure is analogous to subcutaneous injection in humans and does not require anesthesia.

\section{Design and procedure}

The experiment consisted of a handling phase and four phases using the Carousel maze. At the beginning, subjects were handled three days for 5 minutes each day. Next, a 5-day habituation phase followed, during which rats were habituated to the experimental apparatus for 10 minutes each day. The arena was not rotating during the habituation phase. After habituation, subjects were divided into two groups (experimental and control) of 8 subjects such that their locomotion during the habituation phase was similar, but the assignment to groups was random.

The learning phase of the active place avoidance task was 9 days long and one 20 minute session took place during each day for every rat. The session always began during the same 
114 time of a day for each rat. The two groups differed only in arena rotation speed. The speed was

115 always $1 \mathrm{rpm}$ and stable during the whole session for the control group and varied from 0.60 to $1161.34 \mathrm{rpm}$ for the experimental group. Depending on the day, the speed for the experimental

117 group changed every one or two minutes or alternatingly after two and three minutes. The two

118 speeds used for the experimental group during each session were chosen such that the average

119 speed throughout the session was always $1 \mathrm{rpm}$ (i.e. comparable to controls). The change of

120 speed after one minute was used, at most, on two subsequent days to prevent the possible

121 using of the change as temporal information and the speeds were not same any two

122 subsequent days (more detail about rotation speeds can be found on osf.io/683xk/).

123 The following phase (hereafter probe phase) contained one 20 minute long session of the

124 active place avoidance task. A stable rotation speed of $1 \mathrm{rpm}$ was used for both groups during

125 this session. This phase was included to compare avoidance strategies using measures that may

126 be dependent on rotation speed.

127 The last phase of the experiment (dark phase) was three days long and one session of the APA

128 task was scheduled for each day. The sessions were conducted with a stable rotation speed 1

$129 \mathrm{rpm}$ in complete darkness. Subjects were therefore not able to use room orientation cues.

130 Since rats may use not only visual room orientation cues, all possible olfactory cues were

131 removed from the room and three air fresheners were attached to a Plexiglas arena wall to

132 cover possible remaining cues. The Plexiglas itself further hindered use of olfactory and

133 auditory room orientation cues. Additionally, the motor of the apparatus which is positioned

134 under the centre of the arena is a source of loud noise and also prevents possible use of

135 auditory cues. ${ }^{3}$ A summary of the phases of the experiment can be found in Table 1.

Table 1. Phases of the experiment

\begin{tabular}{llll}
\multicolumn{1}{c}{ Phase } & \multicolumn{1}{c}{ Number of sessions } & \multicolumn{2}{c}{ Speed of arena rotation } \\
Habituation & 5 & - & - \\
Learning & 9 & $1 \mathrm{rpm}$ & $0.60-1.34 \mathrm{rpm}$ \\
Probe & 1 & $1 \mathrm{rpm}$ & $1 \mathrm{rpm}$ \\
Dark & $3(4)$ & $1 \mathrm{rpm}$ & $1 \mathrm{rpm}$
\end{tabular}

\section{Apparatus}

137 The Carousel maze (Figure 1) consisted of a smooth metal circular arena $82 \mathrm{~cm}$ in diameter with

138 a low metal rim. Above the rim was a $30 \mathrm{~cm}$ tall transparent Plexiglas wall which enabled easy

139 view outside of the arena. The arena was $1 \mathrm{~m}$ above the ground in a room with a sufficient

\footnotetext{
${ }^{3}$ At the end of the dark phase, one day was added for 6 selected rats that showed some ability to avoid the to-be-avoided sector in the dark. During this day, one session of the APA task in the dark took place to clarify obtained results. The results are described in Supplementary materials on osf.io/z5dny/.
} 
140 amount of visual cues (doors, colored signs on walls, etc.), which served as orientation cues

141 during behavioral testing. On the margin of the arena was a light-emitting diode (LED), which

142 tracked rotation of the arena during the experiment. Another LED was used to track movement

143 of a subject. This LED was fixed to a small metal plate which was attached on the subjects back

144 with two rubber harnesses before each session. A cable for administering electric shocks ran to

145 the metal plate. The cable was attached to a hypodermic needle implanted in a skin fold of the

146 subject with an alligator clip. The position of a rat was tracked during the session with a

147 sampling frequency of $25 \mathrm{~Hz}$ by a computer which was located in the neighboring room. A

148 program used for tracking a rat's position (Tracker 2.33; Biosignal Group, USA) simultaneously

149 on-line evaluated whether the rat was within the to-be-avoided sector and administered a mild

150 electric current in that case. Data were stored for off-line analysis which was conducted with

151 Carousel Maze Manager 0.4.0 [34]. The electric current (AC, $50 \mathrm{~Hz}, 0.5 \mathrm{~s}$ ) was administered

152 whenever the subject entered the to-be-avoided sector for a duration longer than $300 \mathrm{~ms}$. The

153 administered current was initially adjusted to the rat's reaction to elicit response but not a

154 freezing response. All but two subjects responded to $0.4 \mathrm{~mA}$, which was subsequently used for

155 the rest of the experiment. The two mentioned subjects that did not respond to a current of

156 any intensity (maximum used was $0.7 \mathrm{~mA}$ ) were excluded from analysis (the exclusion is further

157 described in Results). Whenever a subject did not escape the to-be-avoided sector within 900

158 ms of the previous shock, another was administered.

\section{Measured parameters}

160 The following parameters were used for subsequent analyses: Total distance was computed as

161 a sum of distances between positions of a subject within an arena (that is without displacement

162 by rotation of the arena) sampled with frequency $1 \mathrm{~Hz}$ and was used to assess locomotion of

163 subjects. Maximum time of avoidance was computed as the maximum duration between two

164 subsequent occurrences of a subject in the to-be-avoided sector. This measure was used to

165 estimate the ability to avoid the to-be-avoided sector. Maximum time of avoidance was equal

166 to $1200 \mathrm{~s}$ when a subject did not get any shock. Maximum time of avoidance usually highly

167 negatively correlates with the number of received shocks. Its distribution is closer to normal

168 and therefore is better suited for analysis of avoidance. Directional mean denotes the average

169 direction of vectors from the center of the arena to subject's position. This can be otherwise

170 described as the direction of a vector obtained from summing unit vectors with directions equal

171 to the direction of a subject relative to the center of the arena. The directional mean may be

172 used to assess strategy of avoidance of the to-be-avoided sector. Circular variance denotes a

173 variability of directions of vectors from the center of the arena to subject's position. It is

174 computed as one minus the length of the vector obtained by summing unit vectors with

175 directions equal to the direction of a subject relative to the center of the arena divided by the

176 number of these vectors. This measure shows to what degree the subject prefers a specific 
position within a room. ${ }^{4}$ Periodicity of movement is computed as the median duration of continuous intervals during which a subject is not moving. It may suggest a strategy used to avoid the to-be-avoided sector. Time in the adjacent sector shows the proportion of time which a subject spent in the sector adjacent to the to-be-avoided sector. The adjacent sector was chosen to be a section of the circle $60^{\circ}$ wide, i.e. of the same width as the to-be-avoided sector. The center of the adjacent sector lay $60^{\circ}$ against the rotation of the arena from the center of the to-be-avoided sector, i.e. the sector from which a subject is moved to the to-beavoided sector in case of immobility. Median speed after shock was computed as the median angular velocity $1 \mathrm{~s}$ after shock which was not followed by another shock sooner than $1 \mathrm{~s}$. Positive median speed after shock shows movement against the rotation of the arena. It may reveal whether a subject moves preferentially against or with the direction of rotation of the arena and with what speed.

\section{Results}

All analyses were done in R 3.0.2 [35]. Analysis scripts as well as data and additional details of procedure are available on osf.io/swxhb/.

\section{Learning phase}

Two subjects that were not able to learn the task were excluded from analysis. These rats were removed from the experiment after the seventh day of the learning phase. Their visibly lower locomotion and maximum time of avoidance can be seen in Figure 2 (see also Figure 4B). Maximum time of avoidance was at a level corresponding to the absence of locomotion - near $50 \mathrm{~s}$, which is a duration between two subsequent presences of a rat in the to-be-avoided sector in case of its immobility (when the arena rotation speed is $1 \mathrm{rpm}$ ).

Analyses were performed with multilevel linear regression with day and group as predictors. Polynomial contrasts were used for the day factor [36]. Only results for linear and quadratic contrasts are reported because higher order contrasts would be hard to interpret. The linear contrast accounts for linearly decreasing or increasing trend and the quadratic contrast

\footnotetext{
${ }^{4}$ For better understanding, directional mean and circular variance can be illustrated using sample tracks in Figure 4. It is possible to see that a subject depicted in Figure $4 \mathrm{C}$ moved within a narrow sector of the arena. Its circular variance is correspondingly .20. On the other hand, the subject depicted in Figure 4B showed little preference for a specific sector and its circular variance is thus .86 (i.e. close to 1). The subject depicted in Figure 4C also moved further away from the center of the arena (in terms of angular movement required for its passive displacement to the to-be-avoided sector by rotation of the arena). Correspondingly, its directional mean is $192.9^{\circ}$, while subjects moving closer to the to-be-avoided sector have lower directional means such as $60.1^{\circ}$ (Figure 4D) and $72.1^{\circ}$ (Figure 4E).
} 
accounts for U-shaped trend in data. In combination they fit well a large pattern of possible results.

Analysis for total distance did not suggest any group effect, $t(12)=0.58, p=.57, r=.16$. Linear contrast for day was not significant, $t(96)=1.37, p=.17, r=.14$, but quadratic contrast was, $t(96)=-2.69, p=.008, r=.26$. The interaction of day and group factors was not significant either for linear, $t(96)=-1.31, p=.19, r=.13$, or quadratic contrast, $t(96)=1.70, p=.09, r=.17$. Therefore, data did not show substantial effect of experimental manipulation on locomotion. This suggests that the task had similar locomotor requirements for both groups. Results for total distance are displayed in Figure 2A.

Maximum time of avoidance during the learning phase did not differ between groups, $t(12)=$ $1.00, p=.34, r=.28$. Change of performance between days was seen in both linear, $t(96)=6.10$, $p<.001, r=.53$, and quadratic contrasts, $t(96)=-3.46, p<.001, r=.33 .{ }^{5}$ Neither the interaction between linear contrast for day and group, $t(96)=-0.80, p=.42, r=.08$, nor between quadratic contrast and group, $t(96)=0.77, p=.44, r=.08$, were significant. This shows that performance of both groups improved during the learning phase (see Figure $4 \mathrm{~A}$ and $4 \mathrm{C}$ for sample behavioral graphs). The difference between groups was seen neither in performance nor in speed of learning. Results for maximum time avoided are depicted in Figure 2B.

\section{Probe phase}

The Wilcoxon test suggested that subjects in the experimental group stayed somewhat further away from the to-be-avoided sector than subjects in the control group, $W=11, p=.10, n_{1}=n_{2}=$ $7, r=.46$. Values of directional means for individual subjects are displayed in Figure $3 \mathrm{~A}$.

Even though a strong correlation of time in the adjacent sector and directional mean suggests that both parameters measure a similar construct, $r_{\mathrm{S}}(12)=-.57, p=.03$, no difference between groups was found for time in the adjacent sector, $W=24, p=1, n_{1}=n_{2}=7, r=.02$. A possible reason may be that values for time in the adjacent sector were low for both groups. Four subjects from the experimental group and three from the control group spent less than $0.5 \%$ of time in the adjacent sector. The absence of difference might have been easily a result of the floor effect. Values of time in the adjacent sector are depicted in Figure 3B.

\footnotetext{
${ }^{5} \mathrm{~A}$ model defined by significant independent variables (intersection with ordinate, linear and quadratic contrasts for day) shows improvement during time which was initially faster, was subsequently getting slower, and reached a peak during the seventh day (prediction of a model is $1021 \mathrm{~s}$ ). However, from the fifth to the last day, the predicted maximum time of avoidance is within a narrow range 951-1021 s. Attainment of this relatively stable level can be seen in Figure 2B.
} 
231 The Wilcoxon test showed no difference in circular variance between groups, $W=30.5, p=.48$,

$232 n_{1}=n_{2}=7, r=-.21$. Circular variance for both groups can be seen in Figure 3C.

233 The Wilcoxon test for periodicity of movement did not reveal any effect of experimental 234 manipulation, $W=24.5, p=1, n_{1}=n_{2}=7, r=0$. Periodicity of movement in the probe phase 235 correlated significantly with circular variance, $r_{\mathrm{S}}(12)=.73, p=.003$, which confirms that 236 periodicity of movement and circular variance measure a similar characteristic of subject's

237 behavior in the task. Higher periodicity of movement means a longer distance that the subject 238 moves by arena rotation during its inactivity. Since subjects usually move only in a certain 239 sector within a room, subjects correct their movement caused by the arena rotation by 240 returning to the position where their inactivity began. Higher periodicity of movement thus 241 causes subject's presence in a wider arena sector within the room frame and therefore leads to 242 higher circular variance as well. The association between periodicity of movement and circular variance indicates validity of both these measures and supports their usability for testing of specific hypotheses in future research.

\section{Dark phase}

246 Results for maximum time of avoidance can be seen in Figure 2B. The Wilcoxon test did not reveal a significant difference in the average maximum time of avoidance between experimental $(M=153 \mathrm{~s}, S D=90 \mathrm{~s})$ and control $(M=110 \mathrm{~s}, S D=25 \mathrm{~s})$ groups, $W=18, p=.44, n_{1}$ $=n_{2}=7, r=.22$. Higher mean and larger variability of values of the experimental group are caused primarily by performance of one subject (hereafter referred as rat 15 ) with the average maximum time of avoidance 354 s. ${ }^{6}$

Although performance during the dark phase worsened considerably in comparison to performance in learning and probe phases, some ability to avoid the to-be-avoided sector was visible even in the dark phase (Figure 4D). A half of the subjects had at least one value of maximum time of avoidance higher than $175 \mathrm{~s}$. Since an absence of locomotion leads to maximum time of avoidance $50 \mathrm{~s}$, these values show that the subjects had to actively avoid the to-be-avoided sector for, at least, two minutes. The highest measured value of maximum time of avoidance was for rat 15 , that was able to avoid the to-be-avoided sector during the third day of the dark phase for $646 \mathrm{~s}$, more than half of the duration of the session (Figure 4E).

260 Even though rats were somewhat able to avoid the to-be-avoided sector, their performance did 261 not improve during the three sessions. Subtracting maximum time of avoidance for the first day from the value for the third day results in positive values only for 5 out of 14 subjects. The

\footnotetext{
${ }^{6}$ The mean and standard deviation for maximum time of avoidance of the experimental group are 120 $\mathrm{s}$ and $19 \mathrm{~s}$ without this subject.
} 
average of these values was $3 \mathrm{~s}$, which shows that with the exception of rat 15, which improved between the first and the third day by $444 \mathrm{~s}$, rats were not improving during the dark phase.

Although subjects were not generally able to avoid the to-be-avoided sector for an extended period of time, basic avoidance behavior was observed even in this phase. This can be seen from the positive values of median speed after shock (analyzed for both groups together), which were higher than zero for all three days of dark phase, $t \mathrm{~s}(13)>4.11$, $p \mathrm{~s}<.002,24^{\circ} / \mathrm{s}<M \mathrm{~s}$ $<28^{\circ} / \mathrm{s}$. This shows that subjects avoided the to-be-avoided sector predominantly by movement against the direction of rotation of the arena, i.e. in a similar manner they solve the task in light (Figure 4D).

\section{Discussion}

The results did not show an effect of variable arena rotation speed on locomotion or the ability to avoid the to-be-avoided sector in the learning phase. Subjects from both groups were able to successfully learn the task and their performance was relatively stable from the fifth day of the learning phase. Therefore, the experiment does not suggest that stable arena rotation speed helps subjects to learn the task.

If subjects used a temporal strategy for avoidance, we would expect that use of this strategy is easier when arena rotation speed is stable rather than variable. We would therefore expect better performance of the control group. Since no difference in performance between the experimental and control group was found, we can conclude that a temporal strategy is not necessary for solving the task. While we can say that a temporal strategy is not necessary for solving the task, it cannot be conclusively inferred from the results that subjects do not use a temporal strategy. It is possible that subjects in the experimental group compensated for the inability to use a temporal strategy by using a different strategy of avoidance. The result is nevertheless important because it shows that a deficit of interval timing should not by itself lead to worse performance in the task. For example, if it is known that some drug causes deficits in interval timing, the results of this experiment suggests, that its possible effect on performance in the APA task could not be assigned only to this effect of the drug.

Subjects in the experimental group may use two strategies to be safe from being moved into the to-be-avoided sector by faster rotation of the arena if we assume that they move against the rotation of the arena with a certain periodicity. The strategies were assessed in the probe phase. The first possible strategy is to stay further away from the to-be-avoided sector. That ensures that even faster rotation of the arena does not move them into the to-be-avoided sector during the period of inactivity. Consistently, a higher directional mean was seen for the experimental group, which suggests that the subjects moved in positions further from the to- 
297 be-avoided sector. It should be noted that while this result was hypothesized, it did not reach

298 statistical significance and is based on a small sample of subjects, so caution with regards to 299 conclusions from it is warranted. The second possible strategy is to move with a lower

300 periodicity. This strategy would enable subjects in the experimental group to adjust their

301 position in the room more often and would again prevent them from being moved into the to-

302 be-avoided sector even during faster arena rotation speed. However, we found no difference

303 which would suggest employment of this strategy in circular variance and periodicity of

304 movement between the groups. It is possible that the rats from the experimental group used a

305 strategy indicated by higher directional mean, i.e. they avoided being moved into the to-be-

306 avoided sector by being further away from it and not by moving within a narrower sector,

307 which would be suggested by a difference in circular variance or periodicity. Both strategies are

308 not mutually incompatible, but using one of them may be sufficient for successful avoidance of

309 the to-be-avoided sector even when the speed of arena rotation is higher.

310 In addition to suggesting a strategy used for avoidance of the to-be-avoided sector, the results

311 of the probe phase showed the possibility of using new parameters to assess specific

312 hypotheses about the influence of experimental manipulations on behavior in the task. From a

313 positive correlation between circular variance and periodicity of movement, it can be seen that

314 both parameters measure a similar construct which partially validates both parameters.

315 Similarly, a negative correlation between directional mean and time in the adjacent sector

316 suggests a convergent validity of both parameters.

317 Since allothetic avoidance cannot be used in dark, any possible difference between groups in

318 the dark phase may reveal the effect of manipulation on the use of alternative strategies of

319 avoidance. However, the results of the dark phase did not suggest any difference between the

320 experimental and control group. Both groups considerably worsened in comparison to the

321 probe phase. Nevertheless, maximum time of avoidance and median speed after shock showed

322 that subjects were still able to solve the task to a certain degree. Movement against the

323 direction of arena rotation, which keeps a subject outside of the to-be-avoided sector by

324 correcting its position within a room [7], persisted even in the dark in most of the subjects. The

325 length of this movement could not have been adjusted by cues outside of the arena and

326 therefore subjects were often moved by arena rotation into the to-be-avoided sector. With the

327 exception of one subject, we did not observe any evidence of improvement of performance

328 over time. It cannot be ruled out that learning avoidance in dark requires a qualitative change

329 of strategy of avoidance and that we would observe an improvement even in other subjects if

330 they had more time for learning. The general lack of improvement cannot be explained by limits

331 of accuracy of idiothetic orientation because one rat was able to avoid the to-be-avoided sector

332 for more than ten minutes. Idiothetic orientation is therefore sufficient for solving the task in

333 the dark, but it does not seem to be crucial for solving the task in light. Since the subject that 
was able to avoid the to-be-avoided sector in dark was from the experimental group, it seems that solving the task in the dark may be based on a combination of inertial and substratal idiothesis rather than on interval timing and substratal idiothesis. A cue indicating when to be active would thus stem from information about passive movement in space rather than from time that passed since the previous movement. Of course, it cannot be excluded that both of these sources of information are combined during the task in dark. It is also possible that the subject was able to learn a temporal strategy during the probe and the dark phases where the speed of arena rotation was stable. Possible conclusions from the results of the dark phase are necessarily limited by the fact that only one of the subjects was able to reliably avoid the to-beavoided sector [30].

The most important limitation, which restricts reliability of conclusions from the study, is a relatively low number of subjects. It is possible that any absence of difference between groups was caused by small statistical power stemming from a low number of subjects in both groups [37]. However, some results are not limited by this issue. For example, it can be seen that even subjects in the experimental group were able to quickly learn the task in light. Even though it is possible that some difference would be found if the experiment had higher statistical power, it is clear that stable arena rotation speed is not necessary for learning the task. Furthermore, it can be seen that rats can learn the task in the dark, even if only one was able to do so. This finding is consistent with the paper by Fajnerova et. al. [30]. Usefulness of some of the newly presented parameters is also clear from their mutual associations that we observed in the present study.

An additional possible limitation is the relatively small range of speeds of arena rotation in the experimental group. The highest speed was only one third faster than the speed used in the control group. This limited range of speeds was due to the technical characteristics of the experimental apparatus. Although it is possible that a wider range of arena rotation speeds would lead to a difference between groups, it is not clear whether a higher speed of arena rotation would not lead to different effects than those that were studied in the present experiment. The goal of the experiment was not to explore the influence of arena rotation speed itself, but of its variability. The arena rotation speeds that we used varied between each session and the difference between maximum and minimum speed was $0.74 \mathrm{rpm}$. This was considered to be sufficient to make time an invalid cue for when a subject should move.

\section{Acknowledgements}

We would like to thank Michaela Fialová, Jindřich Kalvoda and Antonín Zahálka for their technical assistance and Peter M. Luketic for proofreading. 
368

\section{References}

1. Bures J, Fenton AA, Kaminsky Yu, Rossier J, Sacchetti B, Zinyuk L (1997) Dissociation of exteroceptive and idiothetic orientation cues: Effect on hippocampal place cells and place navigation. Philos Trans R Soc Lond B Biol Sci 352: 1515-1524.

2. Bures J, Fenton AA, Kaminsky Yu, Wesierska M, Zahalka A (1998) Rodent navigation after dissociation of the allocenthric and idiothetic representations of space. Neuropharmacology 37: 689-699.

3. Cimadevilla JM, Kaminsky Yu, Fenton AA, Bures J (2000) Passive and active place avoidance as a tool of spatial memory research in rats. J Neurosci Methods 102: 155-164.

4. Fenton AA, Wesierska M, Kaminsky Yu, Bures J (1998) Both here and there: Simultaneous expressions of autonomous spatial memories in rats. Proc Natl Acad Sci U S A 95: 11493-11498.

5. Stuchlik A, Rehakova L, Rambousek L, Svoboda J, Vales K (2007) Manipulation of D2 receptors with quinpirole and sulpiride affects locomotor activity before spatial behavior of rats in an active place avoidance task. Neurosci Res 58: 133-139.

6. Stuchlik A, Petrasek T, Vales K (2008) Dopamine D2 receptors and alpha1-adrenoceptors synergistically modulate locomotion and behavior of rats in a place avoidance task. Behav Brain Res 189: 139-144.

7. Stuchlik A, Petrasek T, Prokopova I, Holubova K, Hatalova H, Vales K, Kubik S, Dockery, C, Wesierska, M (2013) Place avoidance tasks as tools in the behavioral neuroscience of learning and memory. Physiol Res 62: 1-19.

8. Lobellova V, Entlerova M, Svojanovska B, Hatalova H, Prokopova I, Petrasek T, Vales K, Kubik S, Fajnerova I, Stuchlik A (2013) Two learning tasks provide evidence for disrupted behavioural flexibility in an animal model of schizophrenia-like behaviour induced by acute MK-801: A doseresponse study. Behav Brain Res 246: 55-62.

9. Zemanova A, Stankova A, Lobellova V, Svoboda J, Vales K, Vlcek K, Kubik S, Fajnerova I, Stuchlik A (2013) Visuospatial working memory is impaired in an animal model of schizophrenia induced by acute MK-801: an effect of pretraining. Pharmacol Biochem Behav 106: 117-123.

10. Burghardt NS, Park EH, Hen R, Fenton AA (2012) Adult-born hippocampal neurons promote cognitive flexibility in mice. Hippocampus 22: 1795-1808.

11. Vukovic J, Borlikova GG, Ruitenberg MJ, Robinson GJ, Sullivan RK, Walker TL, Bartlett PF (2013) Immature doublecortin-positive hippocampal neurons are important for learning but not for remembering. J Neurosci 33: 6603-6613. 
12. Prokopova I, Bahnik S, Doulames V, Vales K, Petrasek T, Svoboda J, Stuchlik A (2012) Synergistic effects of dopamine D2-like receptor antagonist sulpiride and beta-blocker propranolol on learning in the Carousel maze, a dry-land spatial navigation task. Pharmacol Biochem Behav 102: 151-156.

13. Rambousek L, Bubenikova-Valesova V, Kacer P, Syslova K, Kenney J, Holubova K, Najmanova V, Zach P, Svoboda J, Stuchlik A, et al. (2011) Cellular and behavioural effects of a new steroidal inhibitor of the $\mathrm{N}$-methyl-D-aspartate receptor $3 \alpha 5 \beta$-pregnanolone glutamate. Neuropharmacology 61: 61-68.

14. Cimadevilla JM, Fenton AA, Bures J (2001) New spatial cognition tests for mice: Passive place avoidance on stable and active place avoidance on rotating arenas. Brain Res Bull 54: 559563.

15. Petrasek T, Prokopova I, Bahnik S, Schonig K, Berger S, Vales K, Tews B, Schwab ME, Bartsch D, Stuchlik A (2014) Nogo-A downregulation impairs place avoidance in the Carousel maze but not spatial memory in the Morris water maze. Neurobiol Learn Mem 107: 42-49.

16. Petrasek T, Prokopova I, Sladek M, Weissova K, Vojtechova I, Bahnik S, Zemanova A, Schonig K, Berger S, Tews B, et al. (2014) Nogo-A-deficient transgenic rats show deficits in higher cognitive functions, decreased anxiety, and altered circadian activity patterns. Front Behav Neurosci 8: 90.

17. Kelemen E, Fenton AA (2010) Dynamic Grouping of Hippocampal Neural Activity During Cognitive Control of two Spatial Frames. PLOS Biol 8: e1000403.

18. Stuchlik A, Rezacova L, Vales K, Bubenikova V, Kubik S (2004) Application of a novel Active Allothetic Place Avoidance task (AAPA) in testing a pharmacological model of psychosis in rats: Comparison with the Morris Water Maze. Neurosci Lett 366: 162-166.

19. Lee $H$, Dvorak D, Fenton AA (2014) Targeting neural synchrony deficits is sufficient to improve cognition in a schizophrenia-related neurodevelopmental model. Front Psychiatry 5: 15.

20. Popp SS, Lei B, Kelemen E, Fenton AA, Cottrell JE, Kass IS (2011) Intravenous antiarrhythmic doses of lidocaine increase the survival rate of CA1 neurons and improve cognitive outcome after transient global cerebral ischemia in rats. Neuroscience 192: 537-549.

21. Abdel Baki SG, Kao H-Y, Kelemen E, Fenton AA, Bergold PJ (2009) A hierarchy of neurobehavioral tasks discriminates between mild and moderate brain injury in rats. Brain Res 1280: 98-106. 
432 22. Haber M, Abdel Baki SG, Grin'kina NM, Irizarry R, Ershova A, Orsi S, Grill RJ, Dash P, Bergold 433 PJ (2013) Minocycline plus $\mathrm{N}$-acetylcysteine synergize to modulate inflammation and prevent 434 cognitive and memory deficits in a rat model of mild traumatic brain injury. Exp Neurol 249: 435 169-177.

436 23. Blahna K, Svoboda J, Telensky P, Klement D (2011) Inertial stimuli generated by arena 437 rotation are important for acquisition of the active place avoidance task. Behav Brain Res 216: 438 207-213.

439 24. Kubik S, Stuchlik A, Fenton AA (2006) Evidence for hippocampal role in place avoidance 440 other than merely memory storage. Physiol Res 55: 445-452.

441 25. Wallace DG, Marting MM, Winter SS (2008) Fractioning dead reckoning: Role of the 442 compass, odometer, logbook, and home base establishment in spatial orientation.

443 Naturwissenschaften 95: 1011-1026.

444 26. Mauk MD, Buonomano DV (2004) The neural basis of temporal processing. Annu Rev 445 Neurosci 27: 307-40.

446 26. Mittelstaedt ML, Mittelstaedt H (1980) Homing by path integration in a mammal. 447 Naturwissenschaften 67: 566-567.

448 27. Buhusi CV, Meck WH (2005) What makes us tick? Functional and neural mechanisms of 449 interval timing. Nat Rev Neurosci 6: 755-65.

450 29. Klement D, Levcik D, Duskova L, Nekovarova T (2010) Spatial task for rats testing position 451 recognition of an object displayed on a computer screen. Behav Brain Res 207: 480-489.

452 30. Fajnerova I, Kenney J, Lobellova V, Okrouhlicova S, Stuchlik A, Klement D (2014) Can rats 453 solve the active place avoidance task without the room-bound cues? Behav Brain Res 267: 12645432.

455 31. Coull JT, Cheng R-K, Meck WH (2011) Neuroanatomical and neurochemical substrates of 456 timing. Neuropsychopharmacology 36: 3-25.

457 32. Balci F, Meck WH, Moore H, Brunner D (2009) Timing deficits in aging and neuropathology. 458 In Bizon JL, Wood A, editors. Animal models of human cognitive aging. Totowa, NJ: Humana 459 Press. pp. 161-201

460 33. Mittelstaedt ML, Glasauer S (1991) Idiothetic navigation in gerbils and humans. Zool Jahrb 461 Abt Allgemeine Zool Physiologie Tiere 95: 427-435. 
34. Bahník Š (2014). Carousel Maze Manager (Version 0.4.0) [Software]. Available from https://github.com/bahniks/CM_Manager_0_4_0

35. R Development Core Team (2011) R: A language and environment for statistical computing. R Foundation for Statistical Computing, Vienna, Austria.

36. Baguley, T. (2012). Serious stats: A guide to advanced statistics for the behavioral sciences. Houndmills, England: Palgrave Macmillan.

37. Button KS, loannidis JPA, Mokrysz C, Nosek BA, Flint J, Robinson ESJ, Munafò MR (2013) Power failure: Why small sample size undermines the reliability of neuroscience. Nat Rev Neurosci 14: 365-376.

\section{Figures}

Figure 1. A photograph of the Carousel apparatus. The apparatus consists of a metallic disk which can be rotated at various speeds and directions. A rat is connected with a wire to the swivel on the ceiling. The wire supplies the LED on the rat and delivers mild footshocks (0.2-0.7 $m A)$.

\section{Figure 2. Performance parameters}

Crosses denote mean values for a given group and session (two excluded subjects depicted by empty circles are not included in the mean). Abbreviations: LP - Learning phase, PP - Probe phase, DP - Dark phase

A. Total distance in meters. It can be seen that both groups did not show any difference in locomotion during learning and probe phases. Lower locomotion can be seen in one subject from the control group only during the first two days. However, it attained the level of other subjects by the third session. Absence of a difference between groups in total distance suggests that the experimental manipulation did not cause a higher requirement on locomotion in the experimental group.

B. Maximum time of avoidance in seconds. Maximal possible maximum time of avoidance was equal to the duration of a session, i.e. $1200 \mathrm{~s}$, which corresponds to an absence of entrances in the to-be-avoided sector. Five subjects from each group attained this time during the probe phase, which shows that subjects from both group learned the task well during the learning phase. It can be seen that there was no reliable difference between groups during the learning and probe phases. The horizontal dashed line shows $50 \mathrm{~s}$, which corresponds to the maximum time of avoidance of a non-moving subject (for a speed of arena rotation $1 \mathrm{rpm}$ ). It is clear that 
both subjects that were excluded from analysis were not able to actively avoid the to-beavoided sector. A large decrease in performance is visible for all subjects during the dark phase. One subject from the experimental group was able to avoid the to-be-avoided sector for $646 \mathrm{~s}$ during the third session of the dark phase.

\section{Figure 3. Strategies parameters}

Crosses denote mean values for a given group and session (two excluded subjectsdepicted by empty circles are not included in the mean). Abbreviations: LP - Learning phase, PP - Probe phase, DP - Dark phase

A. Directional mean. Possible values of directional mean range from $0^{\circ}$ to $360^{\circ}$. The value of $0^{\circ}$ and $360^{\circ}$ correspond to the same direction. The to-be-avoided sector is indicated by the dashed line and spans the values of $0^{\circ}$ to $60^{\circ}$. The arena rotation was towards the lower values, which means that larger values of directional mean show that a subject was further away from the tobe-avoided sector. A somewhat higher average directional mean for the experimental group than for the control group can be seen during the probe phase. Five subjects with the highest values of directional mean in this phase were from the experimental group. The higher directional mean suggests that subjects in the experimental group used a safe strategy which was based on a preference of places more distant from the to-be-avoided sector in terms of time required for movement of the subject to the to-be-avoided sector by arena rotation.

B. Time in the adjacent sector as a proportion to the length of a session. The dashed line shows a proportion of time corresponding to homogenous distribution of presence of a subject in all sectors of the arena, which would be expected during full inactivity of the subject. It can be seen that the two subjects excluded from analysis are close to this line. Time in the adjacent sector decreases during the learning phase and is close to zero for nearly all rats at its end. This shows that subjects do not learn only learn to avoid the to-be-avoided sector, but their presence is getting more sparse in the adjacent sector as well. This behavior may be advantageous if subjects do not form an exact representation of the position of the to-beavoided sector within the room. Avoidance of a wider sector may thus lead to better performance in the task. Large increases in time in the adjacent sector can be seen during the dark phase. Time in the adjacent sector in the dark phase often exceeds values expected in cases of homogenous distribution of presence in all sectors of arena. The reason for this is that subjects avoided the to-be-avoided sector with movement against the direction of rotation, but this movement was often initiated only after the administration of a shock.

C. Circular variance. Circular variance has a range of values from 0 (a subject is present only in one direction with relation to the center) to 1 (homogenous presence of a subject in all 
directions in relation to the center - circular variance reaches this value in the case of an immobile subject). In the text, circular variance is analyzed only for the probe phase where similar values can be seen for both groups. Decreasing circular variance can be seen during the first days of the learning phase. This development show that subjects learn to move within a restricted area of the room during the first few days. While the figure suggests a difference between the experimental and control groups in the learning phase, this difference is hard to interpret because it can be caused by the different speed of arena rotation, and not by any difference in the behavior of subjects. High circular variance can be seen in subjects excluded from analysis. It stems from an absence of active avoidance of the to-be-avoided sector. Low circular variance of one subject in the experimental group on the second day of the dark phase can be ascribed to its reaction to the movement into the to-be-avoided sector, which consisted of a short movement against rotation of the arena. Since the rat traveled only a short distance this way, it moved within a narrow sector of the arena which was near the to-be-avoided sector. This is also the cause of its 68 entrances into the to-be-avoided sector and $78.3 \%$ of time in the adjacent sector (see Figure 3B).

\section{Figure 4. Sample angular position graphs}

The graphs present positions of subjects in relation to the to-be-avoided sector (shown by red horizontal lines) during a given sessions. Animals tend to move at the periphery of the arena which means that the angular position displayed in the figure is usually sufficient to represent the exact position of a subject within a room. Only rarely do they move in the center of the arena (indicated by wheat color of vertical bars). Subjects are immobile for most of the session (white) and their displayed movement during this time is only due to the rotation of the arena. The to-be-avoided sector is usually avoided by movement against the direction of the rotation (light green) and when subjects receive a shock (shown as red ticks below a graph) they tend to leave the sector in the same direction (dark green). Movement in the direction of arena rotation (light blue) is present in the initial sessions (A), but usually disappears in the subsequent sessions. The movement in the direction of arena rotation is sometimes used to escape the to-be-avoided sector after a shock (dark blue) especially in subjects who do not learn to actively avoid the sector (B). In the initial sessions, subjects sometimes do not react to a shock by active movement (crimson).

A. First session of the learning phase. The subject did not have any experience with the task which can be seen from the wide range of displayed behaviors. A sucessful strategy of avoidance predominates at the end of the session. 
563 B. Seventh session of an excluded subject. It can be seen that the subject does not actively

564 avoid the to-be-avoided sector. When taken into the sector by arena rotation, it usually escapes

565 further shocks by movement in the direction of the rotation.

566 C. Ninth session of the learning phase. The subject learned to successfully avoid the to-be-

567 avoided sector for the whole session by movement against the direction of arena rotation.

568 D. Third session of the dark phase of a rat with bad performance. While the subject still moves

569 against the direction of arena rotation, it cannot navigate using external cues, which means that

570 it cannot regulate its position within the room properly to avoid entrance of the to-be-avoided

571 sector. Movement is often initiated only after receiving a shock.

572 E. Third session of the dark phase of a rat with good performance. Rat 15 was able to avoid

573 the to-be-avoided sector for 646 s during the displayed session (4:11-14:57). It can be seen that

574 it was able to avoid the sector better than during the first session of the learning phase (A), but

575 worse than during a later session of the learning phase (C). Its position within the room was not

576 as stable as during the ninth session of the learning phase and it was sometimes regulated only

577 after receiving a shock. 
Figure 1 (on next page)

A photograph of the Carousel apparatus.

The apparatus consists of a metallic disk which can be rotated at various speeds and directions. A rat is connected with a wire to the swivel on the ceiling. The wire supplies the LED on the rat and delivers mild footshocks (0.2-0.7 mA). 
PeerJ Reviewing Manuscript| 


\section{Figure 2 (on next page)}

\section{Performance parameters}

Crosses denote mean values for a given group and session (two excluded subjects depicted by empty circles are not included in the mean). Abbreviations: LP - Learning phase, PP Probe phase, DP - Dark phase A) Total distance in meters. It can be seen that both groups did not show any difference in locomotion during learning and probe phases. Lower locomotion can be seen in one subject from the control group only during the first two days. However, it attained the level of other subjects by the third session. Absence of a difference between groups in total distance suggests that the experimental manipulation did not cause a higher requirement on locomotion in the experimental group. B) Maximum time of avoidance in seconds. Maximal possible maximum time of avoidance was equal to the duration of a session, i.e. $1200 \mathrm{~s}$, which corresponds to an absence of entrances in the to-beavoided sector. Five subjects from each group attained this time during the probe phase, which shows that subjects from both group learned the task well during the learning phase. It can be seen that there was no reliable difference between groups during the learning and probe phases. The horizontal dashed line shows $50 \mathrm{~s}$, which corresponds to the maximum time of avoidance of a non-moving subject (for a speed of arena rotation $1 \mathrm{rpm}$ ). It is clear that both subjects that were excluded from analysis were not able to actively avoid the tobe-avoided sector. A large decrease in performance is visible for all subjects during the dark phase. One subject from the experimental group was able to avoid the to-be-avoided sector for $646 \mathrm{~s}$ during the third session of the dark phase. 
A

PeerJ R Total distarice
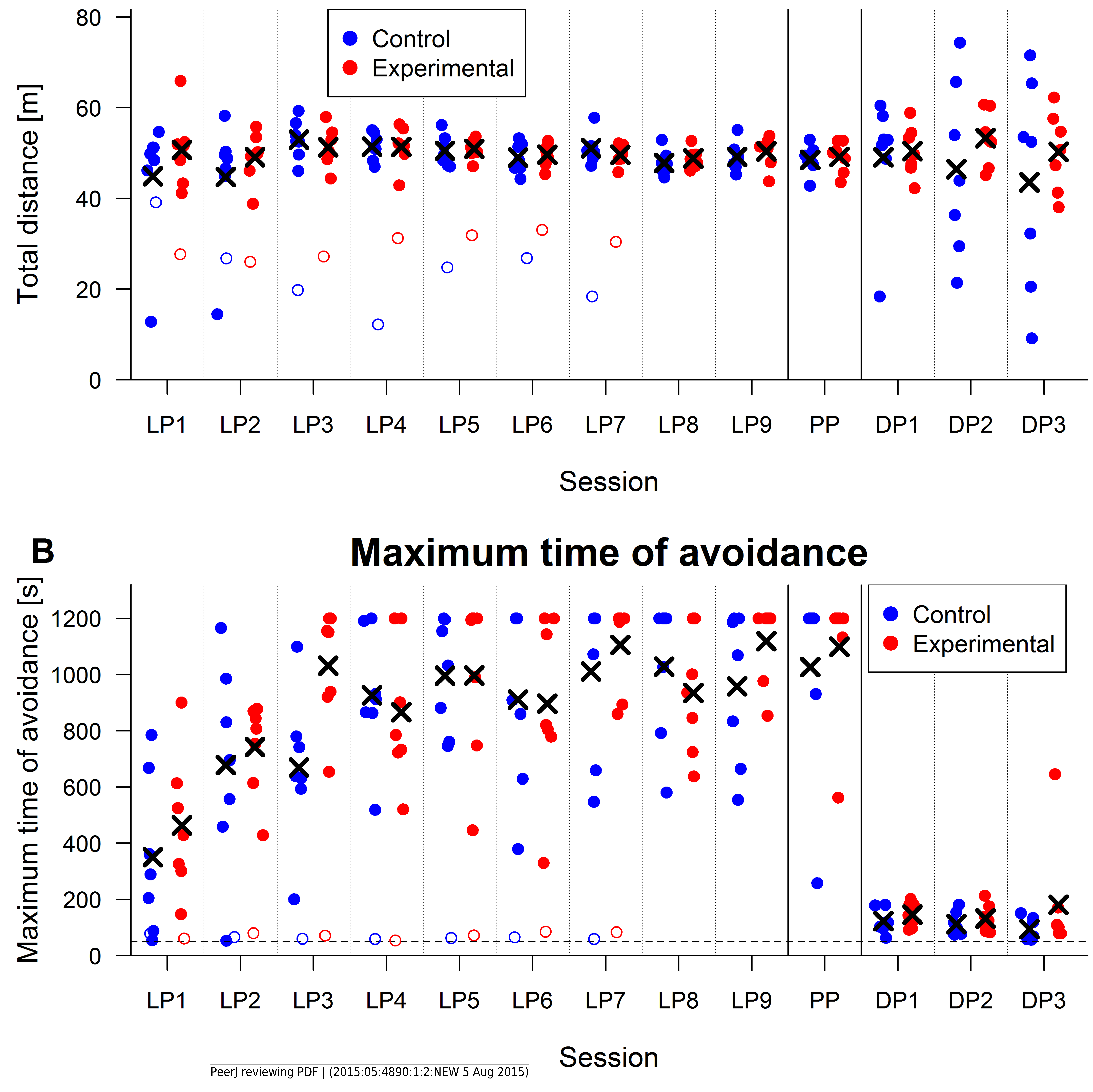


\section{Figure $\mathbf{3}$ (on next page)}

\section{Strategies parameters}

Crosses denote mean values for a given group and session (two excluded subjectsdepicted by empty circles are not included in the mean). Abbreviations: LP - Learning phase, PP Probe phase, DP - Dark phase A) Directional mean. Possible values of directional mean range from $0^{\circ}$ to $360^{\circ}$. The value of $0^{\circ}$ and $360^{\circ}$ correspond to the same direction. The to-beavoided sector is indicated by the dashed line and spans the values of $0^{\circ}$ to $60^{\circ}$. The arena rotation was towards the lower values, which means that larger values of directional mean show that a subject was further away from the to-be-avoided sector. A somewhat higher average directional mean for the experimental group than for the control group can be seen during the probe phase. Five subjects with the highest values of directional mean in this phase were from the experimental group. The higher directional mean suggests that subjects in the experimental group used a safe strategy which was based on a preference of places more distant from the to-be-avoided sector in terms of time required for movement of the subject to the to-be-avoided sector by arena rotation. B) Time in the adjacent sector as a proportion to the length of a session. The dashed line shows a proportion of time corresponding to homogenous distribution of presence of a subject in all sectors of the arena, which would be expected during full inactivity of the subject. It can be seen that the two subjects excluded from analysis are close to this line. Time in the adjacent sector decreases during the learning phase and is close to zero for nearly all rats at its end. This shows that subjects do not learn only learn to avoid the to-be-avoided sector, but their presence is getting more sparse in the adjacent sector as well. This behavior may be advantageous if subjects do not form an exact representation of the position of the to-be-avoided sector within the room. Avoidance of a wider sector may thus lead to better performance in the task. Large increases in time in the adjacent sector can be seen during the dark phase. Time in the adjacent sector in the dark phase often exceeds values expected in cases of

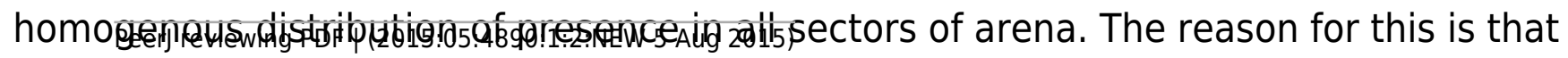


subjects avoided the to-be-avoided sector with movement against the direction of rotation, but this movement was often initiated only after the administration of a shock. C) Circular variance. Circular variance has a range of values from 0 (a subject is present only in one direction with relation to the center) to 1 (homogenous presence of a subject in all directions in relation to the center - circular variance reaches this value in the case of an immobile subject). In the text, circular variance is analyzed only for the probe phase where similar values can be seen for both groups. Decreasing circular variance can be seen during the first days of the learning phase. This development show that subjects learn to move within a restricted area of the room during the first few days. While the figure suggests a difference between the experimental and control groups in the learning phase, this difference is hard to interpret because it can be caused by the different speed of arena rotation, and not by any difference in the behavior of subjects. High circular variance can be seen in subjects excluded from analysis. It stems from an absence of active avoidance of the to-be-avoided sector. Low circular variance of one subject in the experimental group on the second day of the dark phase can be ascribed to its reaction to the movement into the to-be-avoided sector, which consisted of a short movement against rotation of the arena. Since the rat traveled only a short distance this way, it moved within a narrow sector of the arena which was near the to-be-avoided sector. This is also the cause of its 68 entrances into the to-beavoided sector and $78.3 \%$ of time in the adjacent sector (see Figure 3B). 


\section{Figure 4 (on next page)}

Sample angular position graphs

The graphs present positions of subjects in relation to the to-be-avoided sector (shown by red horizontal lines) during a given sessions. Animals tend to move at the periphery of the arena which means that the angular position displayed in the figure is usually sufficient to represent the exact position of a subject within a room. Only rarely do they move in the center of the arena (indicated by wheat color of vertical bars). Subjects are immobile for most of the session (white) and their displayed movement during this time is only due to the rotation of the arena. The to-be-avoided sector is usually avoided by movement against the direction of the rotation (light green) and when subjects receive a shock (shown as red ticks below a graph) they tend to leave the sector in the same direction (dark green). Movement in the direction of arena rotation (light blue) is present in the initial sessions (A), but usually disappears in the subsequent sessions. The movement in the direction of arena rotation is sometimes used to escape the to-be-avoided sector after a shock (dark blue) especially in subjects who do not learn to actively avoid the sector (B). In the initial sessions, subjects sometimes do not react to a shock by active movement (crimson). A) First session of the learning phase. The subject did not have any experience with the task which can be seen from the wide range of displayed behaviors. A sucessful strategy of avoidance predominates at the end of the session. B) Seventh session of an excluded subject. It can be seen that the subject does not actively avoid the to-be-avoided sector. When taken into the sector by arena rotation, it usually escapes further shocks by movement in the direction of the rotation. C) Ninth session of the learning phase. The subject learned to successfully avoid the to-be-avoided sector for the whole session by movement against the direction of arena rotation. D) Third session of the dark phase of a rat with bad performance. While the subject still moves against the direction of arena rotation, it cannot navigate using external cues, which means that it cannot regulate its position within the room properly to avoid entrance of

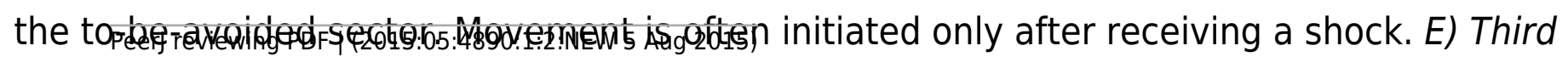


session of the dark phase of a rat with good performance. Rat 15 was able to avoid the to-beavoided sector for $646 \mathrm{~s}$ during the displayed session (4:11-14:57). It can be seen that it was able to avoid the sector better than during the first session of the learning phase (A), but worse than during a later session of the learning phase (C). Its position within the room was not as stable as during the ninth session of the learning phase and it was sometimes regulated only after receiving a shock. 


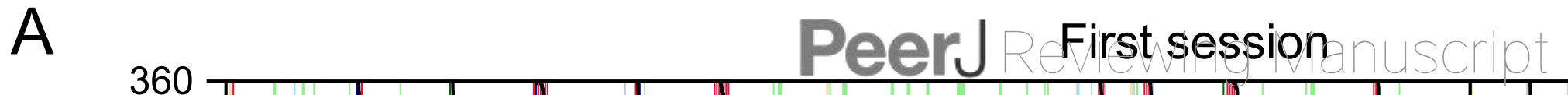

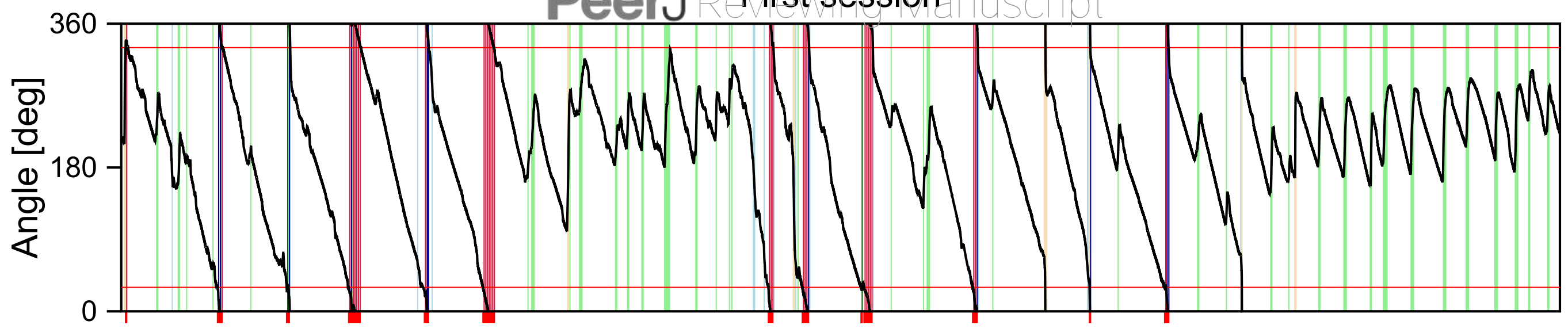

B

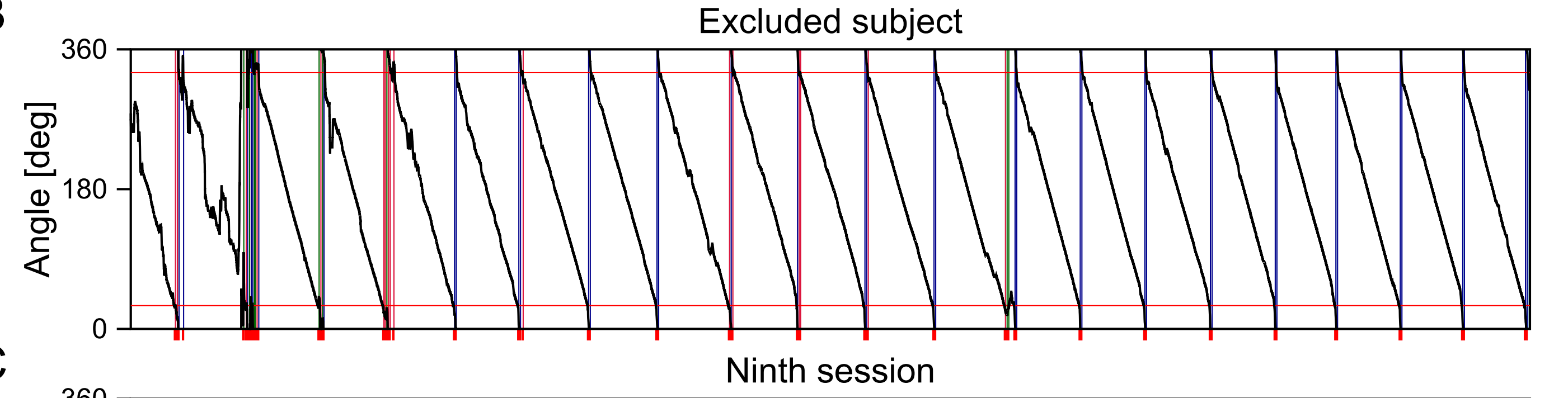

送

D
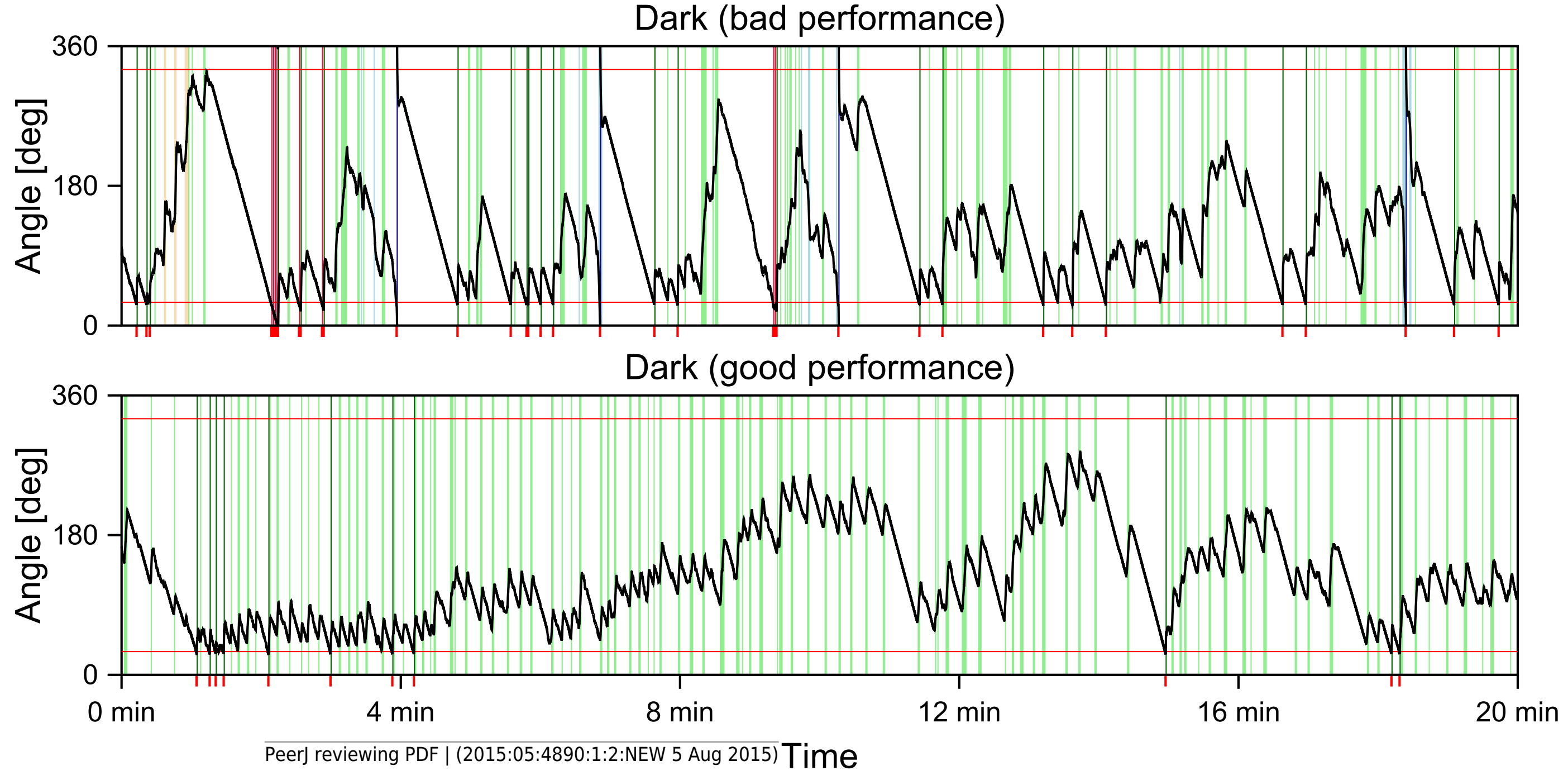
. 